\title{
Wavelet Analysis of the Temporal Dynamics of the Laser Speckle Contrast in Human Skin
}

\author{
Irina Mizeva, Viktor Dremin, Elena Potapova, Evgeny Zherebtsov, Igor Kozlov, Andrey Dunaev
}

\begin{abstract}
Objective: Spectral analysis of laser Doppler flowmetry (LDF) signals has been widely used in studies of physiological vascular function regulation. An alternative to $L D F$ is the laser speckle contrast imaging method (LSCI), which is based on the same physical principle. In contrast to LDF, LSCI provides non-scanning full-field imaging of a relatively wide skin area and offers high spatial and temporal resolutions, which allows visualization of microvascular structure. This circumstance, together with a large number of works which had shown the effectiveness of temporal LSCI analysis, gave impetus to experimental studies of the relation between LDF and LSCI used to monitor the temporal dynamics of blood flow. Methods: Continuous wavelet transform was applied to construct a timefrequency representation of a signal. Results: Analysis of 10 minute LDF and LSCI output signals recorded simultaneously revealed rather high correlation between oscillating components. It was demonstrated for the first time that the spectral energy of oscillations in the $0.01-2 \mathrm{~Hz}$ frequency range of temporal LSCI recordings carries the same information as the conventional LDF recordings and hence it reflects the same physiological vascular tone regulation mechanisms. Conclusion: The approach proposed can be used to investigate speckle pattern dynamics by LSCI in both normal and pathological conditions. Significance: The results of research on the influence of spatial binning and averaging on the spectral characteristics of perfusion monitored by LSCI are of considerable interest for the development of LSCI systems optimized to evaluate temporal dynamics.
\end{abstract}

Index Terms-laser speckle contrast imaging, laser Doppler flowmetry, blood microcirculation, oscillations, wavelets.

\section{INTRODUCTION}

AT PRESENT, there are a variety of methods to detect changes in tissue microcirculation in order to study biochemical processes that are tightly related to blood supply disorders. The dynamic light scattering methods are used in biomedical diagnostics for evaluation of blood flow [1]. The most effective methods for microcirculation assessment are laser Doppler flowmetry (LDF) [2] and laser speckle contrast imaging (LSCI) [3].

LDF technique for perfusion temporal monitoring was introduced by Stern in 1974. Since 2000, much attention has been paid to the analysis of blood flow oscillations. It

Manuscript received June XX, 2019. This study was supported by the Russian Science Foundation under project No.18-15-00201.

I. Mizeva is with the Institute of Continuous Media Mechanics, Perm, Russia, and also with the Research \& Development Center of Biomedical Photonics, Orel State University, Orel, Russia (correspondence e-mail: mizeva@icmm.ru).

V. Dremin and E. Zherebtsov are with the Research \& Development Center of Biomedical Photonics, Orel State University, Orel, Russia, and also with the Opto-Electronics and Measurement Techniques Unit, University of Oulu, Oulu, Finland.

E. Potapova, I. Kozlov and A. Dunaev are with the Research \& Development Center of Biomedical Photonics, Orel State University, Orel, Russia. was found that, apart from functional tests, such analysis can be successfully used to study regulatory mechanisms of cutaneous microcirculation. Spectral decomposition of longterm series perfusion records makes it possible to reveal the oscillatory components corresponding to specific physiological mechanisms.

Cardiac frequency bands $(0.6-2 \mathrm{~Hz})$ and respiratory frequency bands $(0.145-0.6 \mathrm{~Hz})$ provide information about the influence of heart rate and thorax movement on the peripheral blood flow. The myogenic mechanism of vascular tone regulation reflects the response of vascular smooth muscle cells to the transmural pressure resulting in the emergence of oscillations of blood flow at frequencies ranged from 0.05 to $0.15 \mathrm{~Hz}$. The neurogenic sympathetic vasomotor activity induces the movement of vessel walls with a frequency of 0.02 to $0.05 \mathrm{~Hz}$. Slow blood flow waves $(0.005-0.0095$ and $0.0095-0.02 \mathrm{~Hz}$ ) indicate the vascular tone regulation due to the endothelium activity, both nitric oxide-dependent and independent. These mechanisms are considered in detail in Refs. [4], [5], [6], [7]. So, useful diagnostic information is contained in various spectral bands and therefore a timefrequency signal analysis is of high importance.

The LDF technique is based on the measurement of temporal fluctuations of speckle with high time resolution coupled with the subsequent analysis of the power spectrum of intensity fluctuations. The method requires a temporary sample resolution of more than $20 \mathrm{kHz}$. The high data bandwidth limits measurements to just a few points in space precluding fast imaging with substantial both spatial and temporal resolution. Based on the LDF technique, laser Doppler perfusion imaging was developed to enable blood perfusion visualization with spatial resolution diagnostic depth sufficient for skin imaging [8], [9]. However, the scanning process implemented in such systems significantly impairs the temporal resolution of the method limiting the frame rate to a few frames per minute. Another approach involves the use of high-speed cameras [10], [11]. This emerging technology still can be associated with such problems as the high cost of equipment, low spatial resolution and increased noise of detectors due to the tradeoff between frame exposition time and maximum allowed laser irradiance on the skin surface, which results in the low signalto-noise ratio (SNR) of the reconstructed blood flow map.

The LSCI method was originally introduced as a simple and efficient approach for full-field imaging of blood flow, allowing the retrieving of information about the structures involved in the formation of a blood flow signal. LSCI can display the time variation of the speckle at a frequency of several tens of $\mathrm{Hz}$. Thus, it is possible to obtain information 
simultaneously from all pixels with sufficient spatial resolution. LSCI is an effective method for full-scale monitoring of particle dynamics in heterogeneous media [12], [13], [14]. In LSCI, the image of dynamic inhomogeneities is obtained by analyzing the local speckle contrast in the image plane [15], [16]. If a scattering object is illuminated by the coherent light, the randomly changing light intensity pattern, produced by random interference inside the medium and on its surface, appears. This granular structure of light intensity is widely known as the speckle pattern. Movement of particles inside the illuminated medium causes the fluctuations in the position of speckles and results in a blurred image due to the averaging during the exposure time of the detector. Further, temporal and spatial statistics of the speckle pattern can be used for obtaining information on the motion of scattering particles.

It has been demonstrated that the blood perfusion can be estimated both from LDF and LSCI, and these values can be transformed from one to another [17] for the nonhomogeneous scattering medium.

A number of studies have shown that blood perfusion can be assessed by both LDF and LSCI [18], [19], [20]. Assuming only single shifts with a certain speed of the red blood cells and based on the multilayered skin tissue model with certain geometrical and optical properties, blood concentration, and speed distribution given in the theoretical overview [17], it was shown that the LDF power spectrum and the LSCI contrast could be calculated from an optical Doppler spectrum containing various degrees of Doppler-shifted light. The authors provided numerical evidence that a single shifted optical Doppler spectrum can be calculated analytically for a given speed distribution and scattering phase function.

In [21], [22], [23], a comparative analysis of LDF and LSCI methods for assessing cutaneous and cerebral blood flow was carried out. The magnitudes of relative perfusion changes measured by LSCI and LDF show a strong correlation [24], because LSCI and LDF techniques are based on the same physical phenomena of the optical Doppler shift and dynamical light scattering [19], [17].

Despite obvious advantages and active research, only a few studies on human skin microcirculation conducted with the LSCI method have real clinical significance. Most of the works on LSCI image processing are devoted to theoretical issues of processing raw speckle images [25], [26] or cerebral applications [27], [28], whereas the linear and nonlinear analysis of the main microvascular regulatory mechanisms developed for the LDF method could also benefit LSCI signal studies. This has been confirmed in [29] using an empirical mode decomposition analysis of LSCI and LDF signals variability.

As far as we know, the applicability of spectral analyses of LSCI temporal samples to the determination of the effect of different physiological mechanisms has not been justified yet [30]. However, such an approach could provide clinicians with a new imaging tool to extract valuable physiological information about skin blood flow oscillations and its physiological interpretation based on the experience of using LDF measurements [31].

In this work, we employ two methods, LSCI and LDF , in order to analyze the behavior of oscillating cutaneous blood flow components. LDF is used as a reference method [32] and LSCI as a tool for investigating speckle pattern dynamics. Continuous wavelet transform is applied to construct a timefrequency representation of a signal. Wavelets are highly effective for analyzing noisy data and are also useful for correlation analysis of variations in a pair of signals [33].

\section{MATERIALS AND METHODS}

\section{A. Experimental setup}

The scheme of the experimental setup is presented in Fig. 1. The object under study was illuminated by a 10 $\mathrm{mW}$ laser source operating at $635 \mathrm{~nm}$ wavelength (Edmund Optics Inc., USA). A CMOS-camera DCC 3260M (Thorlabs, Inc., USA) with $1936 \times 1216$ pixels and $5.86 \mu \mathrm{m}$ pixel size, camera lens MVL25M23 (Thorlabs, Inc., USA) and a polarizer with its polarization axis perpendicular to the illumination polarization were used to record raw speckle images. The polarizer rejected specular reflections from the skin surface. The distance between the camera lens and the area of interest was $25 \mathrm{~cm}$. The typical raw speckle map is presented in Fig. 2 . For maximization of the (SNR), the minimal speckle size must exceed the Nyquist criterion [34]. Thus, the speckle size on the camera was adjusted by changing the pupil diameter of a camera lens to achieve a speckle size at least 2 times the pixel size. The speckle size is estimated using equation [34]: $S=2.44 \lambda(1+M) f / \#$, where $\lambda$ is the illumination wavelength, $M$ is the imaging system magnification, and $f / \#$ is the camera lens aperture.

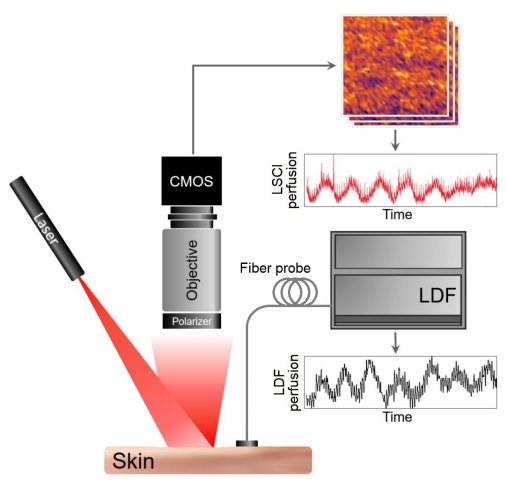

a)

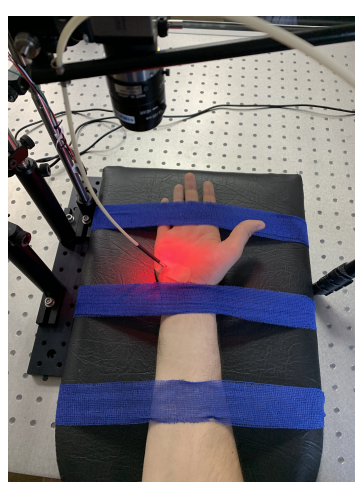

b)
Fig. 1. Scheme of the experimental setup (a) and its overview (b). The LSCI signal presented in the figure was obtained after averaging over the whole image.

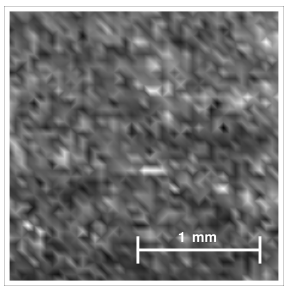

a)

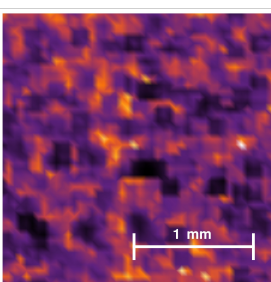

b)

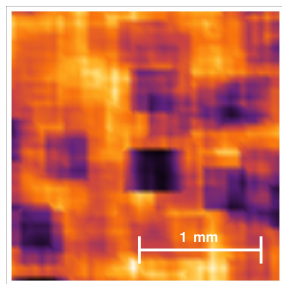

c)
Fig. 2. (a) Instantaneous speckle image, contrast images $1 / \sigma_{N}$ for (b) $N=3$ and (c) $N=7$. Dark spots correspond to minimum values and maximum light intensity. 
The images obtained were processed with a customdeveloped algorithm using Matlab R2018b. The average speckle contrast of the image was calculated Eq. 1 [35]:

$$
K=\frac{\sigma_{N}}{\left\langle I_{0}\right\rangle_{N}}
$$

where \langle\rangle is the symbol of averaging, $N$ is the window of averaging $N \times N$, and $\sigma_{N}$ is the standard deviation in the window $N \times N$.

In this study, the LSCI perfusion was calculated as $1 / K^{2}$. Examples of speckle contrast images are presented in Fig. 2(b,c) for $N=3$ and $N=7$, respectively.

Perfusion was also measured by the experimental system "LAKK-02" (SPE "LAZMA" Ltd., Russia). The single-mode laser with $1064 \mathrm{~nm}$ wavelength was utilized as a radiation source. Optical fibers were used to deliver radiation to the skin surface and to collect back scattered light.

\section{B. Data analysis}

The raw data we use here present a temporal sequence of $2 \mathrm{D}$ speckle images. We assume that the manifestation of LSCI perfusion oscillations can depend on the averaging area. At the first stage, we calculated speckle contrast (Eq. 1) for each speckle image in a moving averaging window of $7 \times 7$ pixels. The collected stack of speckle contrast images allowed reconstruction of the temporal dynamics of the parameter at every image point as well as within an averaging region. Using this information, we converted the data into a set of 1D temporal signals varying the square of averaging. We divided the region of interest (ROI) into $A_{1}-25$ squares of $5 \times 5$ and $A_{2}$ 100 squares of $10 \times 10$ matrix elements (see Fig. 3). For every square subregion, the averaged LSCI perfusion was calculated. So, we obtained 25 temporal samples in $A_{1}$ and 100 in $A_{2}$ cases. In addition, we calculated spectral characteristics for every sample over $A_{1}$ and $A_{2}$ in order to evaluate the effects of averaging and the spatial variation of the spectra along a selected direction. Estimating wavelet cross-correlation, we considered 20 pairs of temporal series randomly chosen from the $A_{1}$ and $A_{2}$ regions measured in one typical volunteer. After that, we calculated wavelet correlation for each pair and estimated median, first and third quartiles of the determined distribution. The obtained results were reliably repeated in different series of experiments with various volunteers.

In the next step, we evaluated the relation between LSCI and LDF signals. We considered here only the averaged speckle contrast over the whole ROI (without splitting). Spearman's correlation was used to compare the average values of the two signals. Due to the nonlinear coupling between the features, the lack of data on the distribution and a small number of observations, we had to use non-parametric methods. Further, we performed the spectral analysis of two signals normalized to the square of standard deviation (SD). Comparison with the wavelet cross-correlation analysis was also carried out.

The wavelet transform $W_{x}(\nu, \tau)$ of a signal $x(t)$ is defined in terms of appropriate mother wavelet $\psi(t)$ as given in Eq.2:

$$
W_{x}(\nu, \tau)=\sqrt{\nu} \int_{\infty}^{\infty} x(t) \psi^{*}[\nu(t-\tau)] d t,
$$

where $t$ is the time, $\tau$ is the time shift of the wavelet, $\nu$ is the oscillations frequency, and the symbol * indicates the operator of complex conjugation. We utilize here the wavelet Morlet [36]:

$$
\psi(t)=e^{2 \pi i t} e^{-t^{2} / 2 \sigma^{2}} .
$$

The wavelet Morlet is one of the commonly used wavelets. Being complex, this wavelet allows studying the amplitude and phase properties of oscillations of different frequencies in the signal. This type of wavelet makes it possible to vary temporal-frequency resolution, varying thus the decay parameter. To reduce boundary effects and to increase the number of independent oscillations, we have chosen small numbers of waves in the wavelet, and $\sigma$ is equal to 1.7 [37].

The integrate wavelet spectra are calculated by integrating the squared absolute value of wavelet coefficients over period $T$ :

$$
M(\nu)=\frac{1}{T} \int_{0}^{T}\left|W_{x}(\nu, \tau)\right|^{2} d \tau .
$$

Since all the experimental signals are measured in arbitrary units and the value of the LDF and LSCI value depends on the physiological properties of the skin, we normalize the total power of oscillations with frequency $\nu$ to the square of SD. Thus, we have

$$
E(\nu)=M(\nu) / S D^{2} .
$$

The wavelet cross-correlation of two signals $x(t)$ and $y(t)$ is defined in terms of their wavelet transforms as follows:

$$
C_{x y}(\nu)=\frac{\int_{0}^{T} W_{x}(\nu, \tau) W_{y}^{*}(\nu, \tau) d \tau}{\sqrt{\int_{0}^{T}\left|W_{x}(\nu, \tau)\right|^{2} d \tau \int_{0}^{T}\left|W_{y}(\nu, \tau)\right|^{2} d \tau}} .
$$

The absolute value of $C_{x y}(\nu)$ belongs to the interval of $[0,1]$ and describes the degree of correlation of oscillations at a given frequency $\nu$. The phase shift between oscillations in the pair of signals is dtermined as

$$
\phi(x y)=\arctan \frac{\Im\left(C_{x y}\right)}{\Re\left(C_{x y}\right)} .
$$

\section{Measurement procedure}

Measurements were carried out in a quiet room at $20-24{ }^{\circ} \mathrm{C}$ after pre-adaptation to room conditions. Studies avoided exposure to a strong source of artificial light and sunlight shining on the participant. During the experiments, the subjects were in the sitting position. For each subject, the measurements of speckle contrast and LDF were conducted. The LDF fiber probe was located on the base of the palm, little finger side.

LDF and LSCI samples were collected from the front side of the palm in the middle part of hypothenar eminence (Fig. 1(b)). LDF probe was fixed on the skin surface by the patch with minimal tissue compression [38]. Both methods operated in the area free of large vessels, the ROI for speckle-contrast imaging was chosen at a 2-3 $\mathrm{mm}$ distance from LDF. 

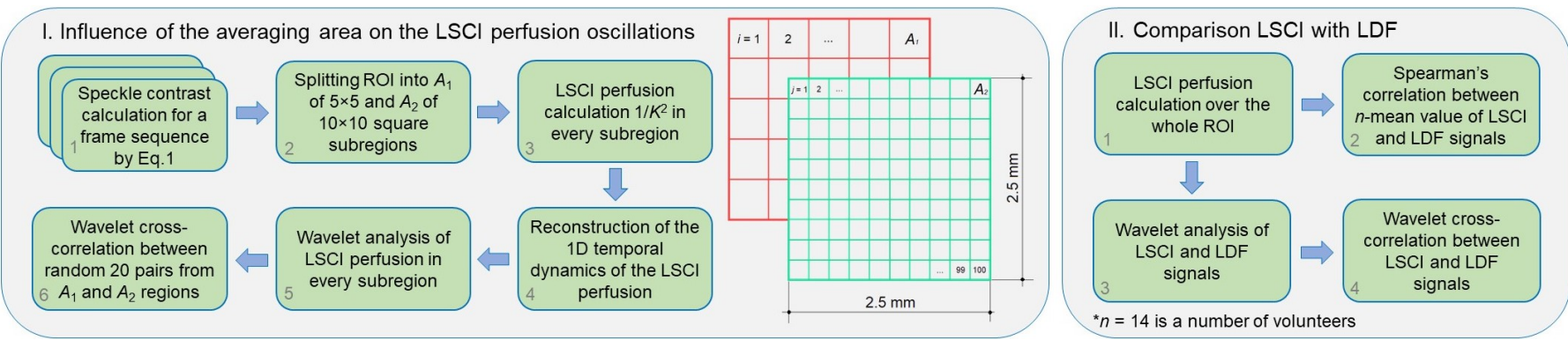

Fig. 3. Data analysis flowchart. Selection of areas for studying the effect of spatial averaging on the spectral characteristics of LSCI perfusion time series is illustrated in the first block to the right.

The LSCI experimental setup was located $25 \mathrm{~cm}$ (as recommended [39], [40]) away from the skin surface followed by adjusting the zoom, focus and polarizer for minimal specular reflection.

The hand was additionally fixed with soft elastic bandages "Peha-haft" (Hartmann, Germany) to the stand to prevent involuntary movements (Fig. 1(b)). The duration of each measurement was 10 minutes. The data was acquired at the sampling frequency of $80 \mathrm{~Hz}$ and the exposure time of $9 \mathrm{~ms}$ via LSCI and $20 \mathrm{~Hz}$ via LDF. Finally, the output data obtained from one simultaneous recording contained 12000 LDF data points and 48000 speckle images.

\section{Subjects}

Fourteen healthy subjects ( 8 males and 6 females) participated in this study. Their median age was 27 years and was within the range of 20 to 42 years. The subjects were caffeine and medications free. The study did not include volunteers with bronchopulmonary, cardiovascular, or neuroendocrine system diseases, nor with diseases of the gastrointestinal tract, liver, kidneys, blood, and any other serious chronic diseases, which could have an impact on the study results. Volunteers with a history of alcoholism, drug addiction, and drug abuse were also excluded. Informed consent was provided by all participants. The study was approved by the ethics committee of Orel State University (Orel, Russia).

\section{RESULTS}

An example of the temporal evaluation of $1 / K^{2}$ along a vertical cross-section in the ROI is presented in Fig. 4. The signal is heterogenic through the spatial dimension, and the waves of $1 / K^{2}$ with the characteristic time close to $10 \mathrm{~s}$ are well seen. Such pulsations with the frequency of about $0.1 \mathrm{~Hz}$ may be associated with the myogenic microvascular activity.

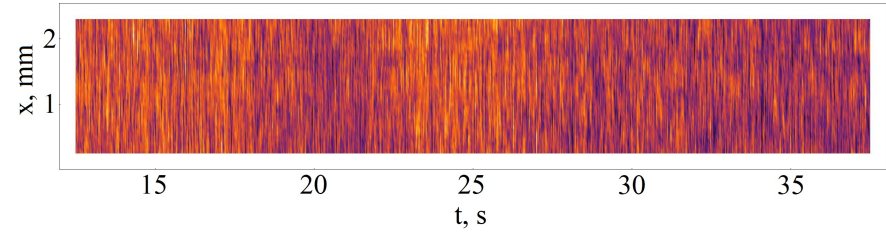

Fig. 4. The evolution of LSCI perfusion $1 / K^{2}$ (a.u.) obtained as a vertical (along $y$ axe in the ROI) cross-section of the spatial-temporal 3D speckle contrast data.
Figure 5 presents typical examples of time evolution obtained for the considered blood perfusion signals. The LDF signal (Fig. 5(a)) demonstrates pronounced oscillations. In the speckle contrast $1 / K^{2}$, almost the same time evolution with quasi-periodical behaviour is observed. Together with the large-scale oscillations whose period is close to $10 \mathrm{~s}$, the signal may have high-frequency periodicity (the period is close to 1 s). Note that the signal characteristics depend on the averaging area. Namely, the noise impact increases significantly with decreasing averaging area (Fig. 5(b,c)).

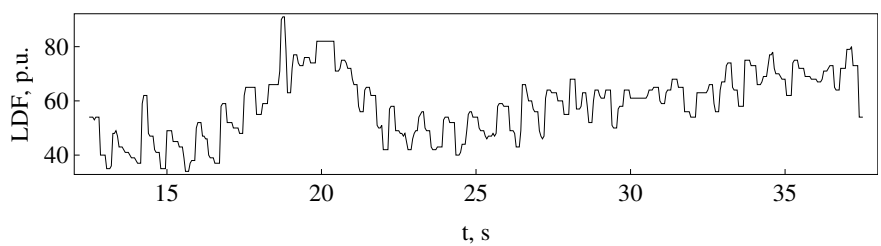

a)

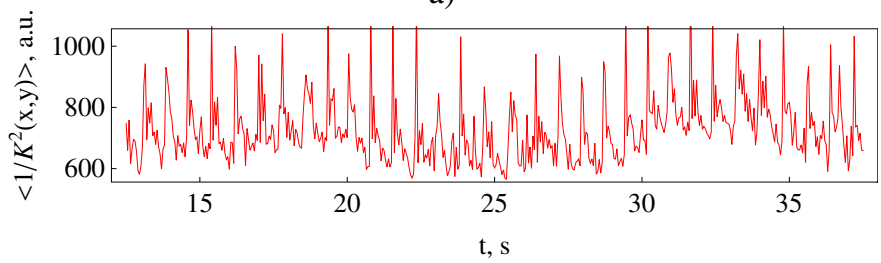

b)

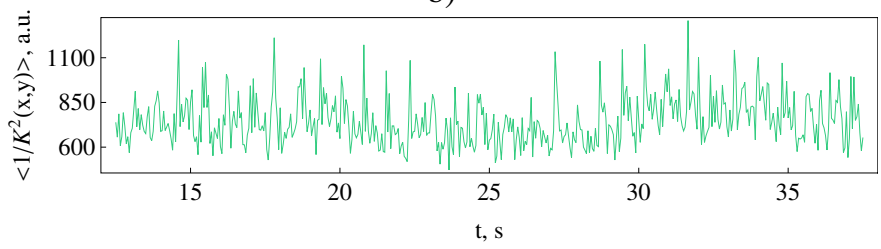

c)

Fig. 5. Typical temporal evolution of perfusion associated signals. (a) LDF signal, (b) LSCI perfusion $1 / K^{2}$ (with $7 \times 7$ computational window) averaged over the $10 \times 10$ matrix elements and (c) over the $5 \times 5$ matrix elements and collected simultaneously via LDF.

Now we turn to the study of the spectral composition of both LDF and spectral contrast time series. For this purpose, we assume that the manifestation of the LSCI perfusion oscillations depends on the averaging area. To reinforce this idea, we have investigated the influence of the averaging parameters, i.e. the spatial variability of spectral characteristics for the obtained time series was estimated. In Fig. 6(a), the mean value of spectral energy for every frequency is shown by 
the thick solid line, and first and third quartiles by the dashed line. Colors indicate the ways of splitting (green is by $5 \times 5$ and red is by $10 \times 10$ elements).

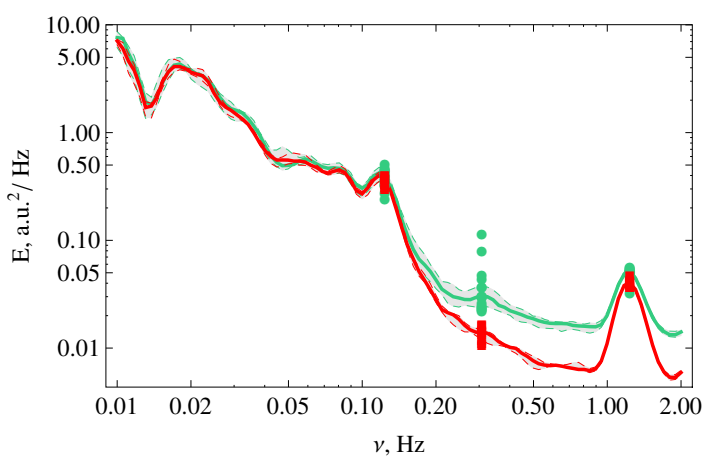

a)

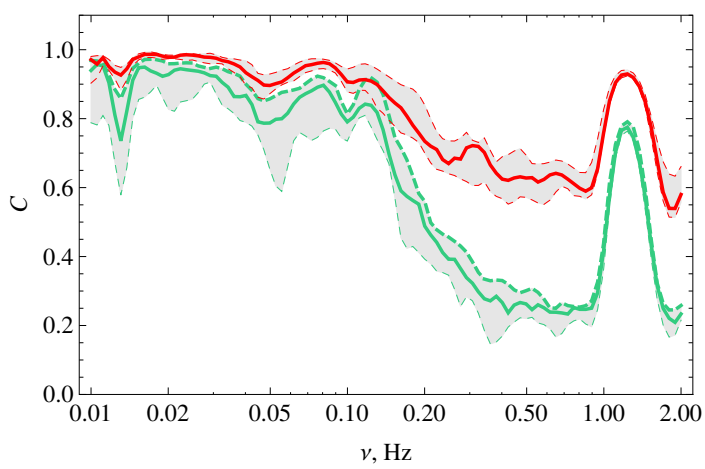

b)

Fig. 6. (a) Typical mean values of the spectra for LSCI temporal recordings (solid lines) with first and third quartiles (dashed lines). Separate points indicate spectral energy of oscillations at certain frequencies obtained from different regions. (b) Wavelet cross-correlation for 20 randomly selected pairs of LSCI perfusion calculated in subregions of $A_{1}$ (green) and $A_{2}$ (red). For both (a) and (b) the colors of the lines correspond to the averaging parameters described for the plots (b) and (c) in Fig. 5, respectively.

First, let us mention that the structures of the spectra calculated by different splitting techniques are similar. All spectra have maxima at several specified frequencies, close to $0.1,0.3$ and $1.3 \mathrm{~Hz}$. It is shown that energy variations in the neighbourhood of these maxima are different. That is 0.1 and $1.3 \mathrm{~Hz}$ pulsations have pronounced maxima with a little divergence and at the same time the $0.3 \mathrm{~Hz}$ oscillations have much higher divergence. To illustrate this hypothesis, we considered spectral energies at certain frequencies $(0.1,0.3$, $1.3 \mathrm{~Hz}$ ) (points in Fig. 6(a)). In the $F$-test, we compared the differences in spectral energy at these frequencies, which were obtained using different splitting techniques to produce a time series. It was established that the variance is the same at 0.1 and $1.3 \mathrm{~Hz}$ and differs significantly at $0.3 \mathrm{~Hz}(\mathrm{p}=0.0002)$. In our opinion, this is indicative of the locality of respiratory oscillations.

We chose 20 random pairs of such time series for $5 \times 5$ and $10 \times 10$ averaging and estimated the statistical properties of cross-correlation characteristics (Fig. 6(b)). It is seen that the $0.1 \mathrm{~Hz}$ and $1.3 \mathrm{~Hz}$ oscillations in different areas are correlated. At the same time, the $0.3 \mathrm{~Hz}$ oscillations have a very weak spatial correlation. We suppose that this says for the spatial heterogeneity of the $0.3 \mathrm{~Hz}$ oscillations as compared to the $0.1 \mathrm{~Hz}$ and $1.3 \mathrm{~Hz}$ oscillations.

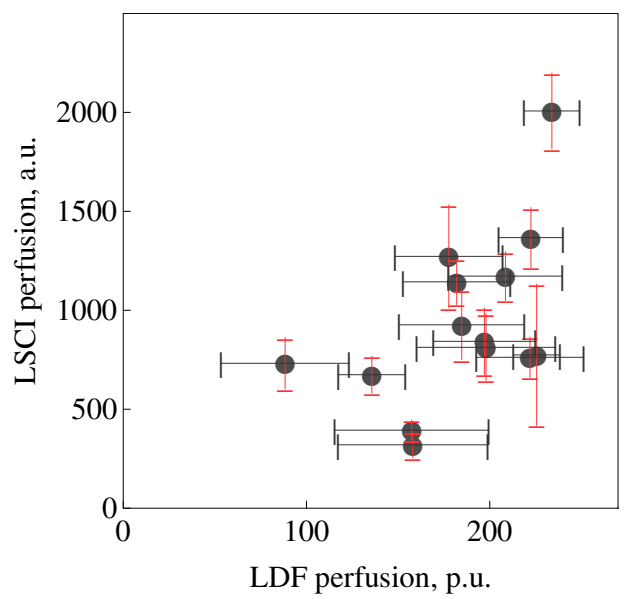

Fig. 7. Ratio of the mean values of blood perfusion measured via LDF and LSCI for the group of 14 healthy volunteers. Black error bars indicate standard deviation of LDF perfusion and red - standard deviation of LSCI perfusion.

It is seen in Fig. 7 that there is a relation between blood the perfusion signals measured via LDF and LSCI.For the group of recruited healthy volunteers, the Spearman correlation between two parameters is $0.60(\mathrm{p}=0.02)$. This correlation value indicates that the relation between these two measures is monotonic. Despite the fact that the obtained coefficient is moderate, we can say, relying on the results of the reliability test, that it is significant.

Next, we consider only the averaging speckle contrast over the whole ROI (without splitting). Having compared the LDF and LSCI spectra, we normalize the spectral energy to the square of SD. The skin perfusion values measured via LDF and LSCI and averaged over the group spectra are presented in Fig. 8. First of all, it should be noted that both spectra have common structure. Close to $1 \mathrm{~Hz}$, the peak associated with the cardiac activity is observed. Also, the low-frequency perfusion oscillations of blood perfusion are detected. The frequencyby-frequency correlation analysis performed using the wavelet cross-correlation demonstrated rather high correlation of lowfrequency oscillations (Fig. 9). In the frequency band 0.01-0.1 $\mathrm{Hz}$, the mean value of cross-correlation varies near $0.7-0.8$, and this correlation turns out to be significant.

The phase shift between the LDF and LSCI signals at frequencies of about $0.4-2 \mathrm{~Hz}$ is close to zero. The frequency band in the neighborhood of $1 \mathrm{~Hz}$ is associated with the cardiac activity, thus we have found that the phase shift of the pulse wave registered by two techniques is zero. Oscillations with frequencies $0.4-0.8 \mathrm{~Hz}$ have very low energy and weak correlation, which leads to high divergence in the phase shift, so the mean value is also close to zero.

At the same time, we have established a significant phase shift in the frequency band $0.02-0.1 \mathrm{~Hz}$ which varies from -0.4 to $-0.6 \mathrm{rad}$. Such a phase shift gives time lag $\approx 4 \mathrm{~s}$ for $0.02 \mathrm{~Hz}$ and $\approx 0.6 \mathrm{~s}$ for $0.1 \mathrm{~Hz}$. This means that the temporal oscillations with frequencies of $0.2-0.1 \mathrm{~Hz}$ in speckle contrast follow the same oscillations in LDF. 


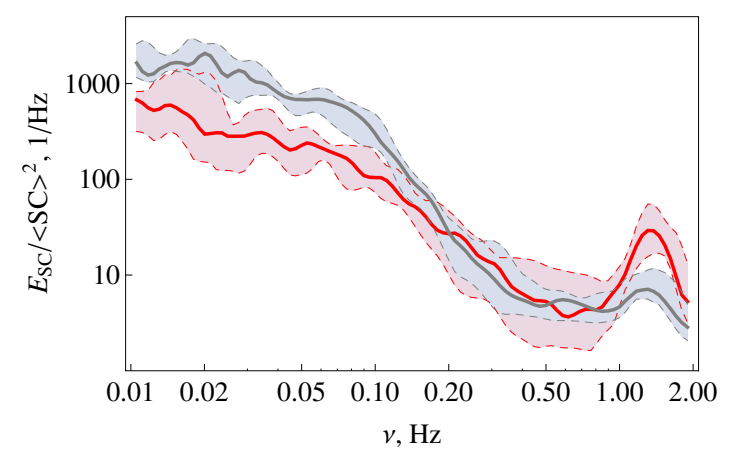

Fig. 8. Averaged over the whole ROI normalized spectra of skin perfusion measured in the group of healthy volunteers using LDF (gray) and LSCI (red). Solid lines indicate median values, and dashed - first and third quartiles.

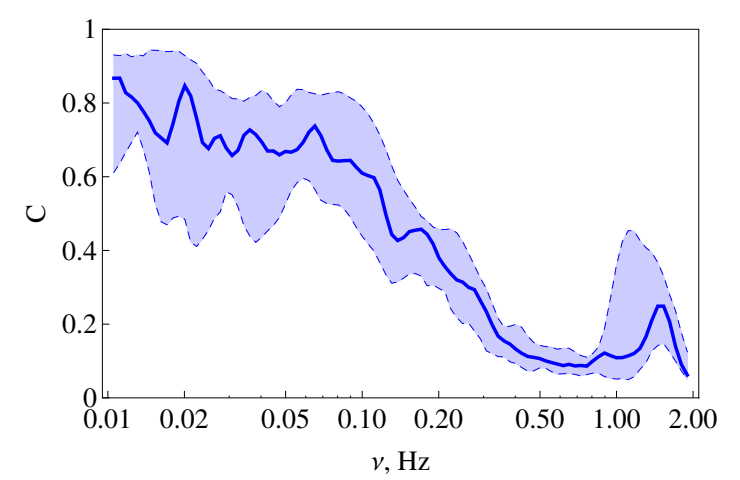

a)

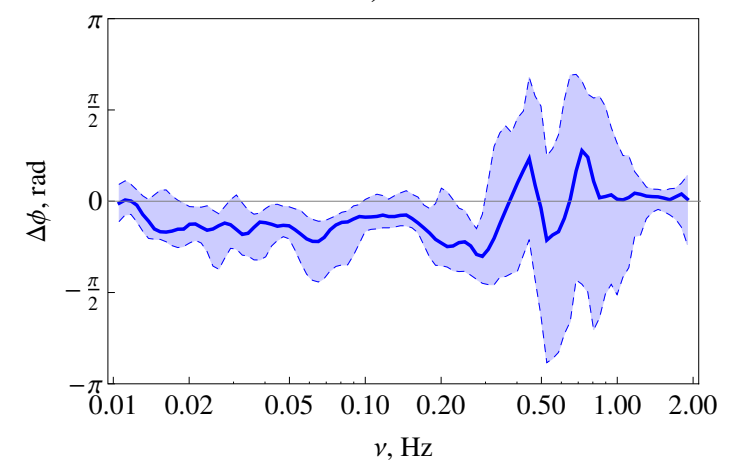

b)

Fig. 9. (a) Absolute value and (b) phase shift of wavelet cross-correlation between LDF and LSCI signals (averaged over the whole ROI) measured in the group of healthy volunteers. Solid lines indicate median values, and dashed - first and third quartiles.

\section{DiscusSION}

The central focus of the study is to answer whether it is possible to identify blood perfusion oscillations via LSCI. For this purpose, we have estimated a correlation between the LDF and LSCI signals using the wavelet-correlation analysis and Spearman's correlation analysis. The main difference between these techniques is that LDF measures perfusion point-wise and with low spatial resolution, whereas LSCI offers an image of blood perfusion. Interestingly, the closest spectra are obtained using an integration over the largest available area. The possible reason for this is the averaging procedure, which reduces the noise level. In [41], this phenomenon was also demonstrated for the averaged LSCI values obtained from the regions of interest which were large enough; multifractal spectra became larger and closer to those of the LDF signals.

The absolute values of the spectral energy are very difficult to compare due to the peculiarities of measuring blood perfusion by both techniques. In particular, LDF and LSCI give blood perfusion in arbitrary units. To compare LDF and LSCI spectra, we considered the spectral energy normalized to SD. Such presentation provides very close spectra, the differences of which can be explained by the characteristics of electronic filters than by physiological factors. Based on the experience gained in the LDF signal analysis, the most effective way is to measure relative variations in perfusion caused by physiological stimuli [42], [38], [43]. We suggest that the same approach can be used in the future studies of LSCI temporal evolution oscillations. The wavelet crosscorrelation analysis has revealed significant coherence, which supports the hypothesis that oscillation generation sources are common. We also note that the pulse wave measured by both techniques is in phase. At the same time, the oscillations assessed by LSCI within the low-frequency band of 0.02-0.1 $\mathrm{Hz}$ lag behind the LDF oscillations with the phase shift close to $0.5 \mathrm{rad}$. We assume that this delay is due to the different depth of light penetration from the sources used in the LSCI and LDF techniques. Speckle technology has more shallow diagnostic depth than the laser Doppler technique since the laser source in the speckle installation is at a considerable distance from the object, and the power density of the laser beam on the skin decreases [44]. It was shown in Ref. [35] that the assessed depths for LDF can be three times higher than those for LSCI. Similar results for the LDF method were obtained in our previous work [45]. Thus, for LCSI technique, photons penetrate the epidermis, papillary dermis, and small part of upper blood net dermis. The LDF probe is highly sensitive to the variations of blood flows in the papillary dermis and upper blood net plexus and is able to cover the top part of the reticular dermis. It should also be pointed out that the sampling volume and the effective probing depth essentially depend on the dynamic range of the detector and specific source-detector configuration.

Figure 7 shows a strong scatter in the data. This is probably due to the large heterogeneity of perfusion values between subjects and palm areas where the LDF and speckle signals were recorded. While measuring, we tried to position the LDF probe and focus of the LSCI setup at the same place for each subject. The Spearman correlation value for our data is nearly consistent with the results published in [30], where the perfusion values were compared using the LSCI and LDF techniques.

\section{CONCLUSION}

In the past two decades, starting with paper [42], the LDF spectral energy in the frequency range from $0.01 \mathrm{~Hz}$ to $2 \mathrm{~Hz}$ was associated with several physiological mechanisms which control the tone of peripheral vessels. Such an approach has been successfully utilized in studies on blood microcirculation in normal and pathological conditions. It provides deeper insight into physiological factors leading to microvascular 
abnormalities in such diseases as diabetes mellitus, rheumatic diseases, etc. Together with advantages that include noninvasive long-term recordings, the LDF technique has some disadvantages associated with the locality of measurements, which is essential due to the significant heterogeneity of a cutaneous vessel network.

LSCI allows one to create a two-dimensional mapping of the perfusion of the desired area with good temporal and spatial resolution at a reasonable cost of installation. This paper was aimed to reveal the possibility of applying the LDF spectral decomposition technique to LSCI data. For the first time, it has been demonstrated that the spectral energy of oscillations in the $0.01-2 \mathrm{~Hz}$ frequency range of the temporal recordings of speckle contrast carries the same information as the conventional LDF recordings and can be associated with the same physiological mechanisms. We have revealed rather high correlation of pulsatile components of LSCI and LDF samples even in the case of mean values demonstrating moderate correlation.

To construct the temporal evolution of LSCI, of primary importance is selection of an optimal area for averaging. When the area of integration increases, the SNR decreases and the LSCI spectrum approaches the LDF spectrum.

We have obtained that the low-frequency blood flow oscillations, which are associated with the active mechanisms of regulating vascular tone and extremely important in the diagnosis of microvascular abnormalities, measured by both techniques, are highly correlated. We mention the stable phase shift of oscillations of the frequencies, which can be explained by the different deepness of bedding the vessels. Summarizing the results obtained, we conclude that the LDF spectral analysis methodology can be extended to LSCI.

This study supports the statement that the approaches for spatial and temporal dynamics evaluation should be applied in accordance with their scope, strengths and weaknesses. Temporal evolution is useful for blood flow monitoring. Spatial structure characterizes the flow with respect to the density and homogeneity of the microvascular network and makes it possible to avoid the proximity of larger arterioles and small arteries.

\section{ACKNOWLEDGEMENTS}

We thank anonymous referees for their comments which helped us to improve the work.

\section{REFERENCES}

[1] V. Tuchin, Handbook of Coherent Domain Optical Methods: Biomedical Diagnostics, Environmental and Material Science, ser. Handbook of Coherent Domain Optical Methods: Biomedical Diagnostics, Environmental and Material Science. Kluwer Academic Publishers, 2004.

[2] V. Rajan, B. Varghese, T. G. van Leeuwen, and W. Steenbergen, "Review of methodological developments in laser doppler flowmetry," Lasers in Medical Science, vol. 24, no. 2, pp. 269-283, 2009.

[3] S. Eriksson, J. Nilsson, and C. Sturesson, "Non-invasive imaging of microcirculation: a technology review," Medical Devices: Evidence and Research, vol. 7, p. 445452, 2014.

[4] H. D. Kvernmo, A. Stefanovska, K. A. Kirkeboen, and K. Kvernebo, "Oscillations in the human cutaneous blood perfusion signal modified by endothelium-dependent and endothelium-independent vasodilators." Microvascular Research, vol. 57, no. 3, pp. 298-309, 1999.
[5] P. Kvandal, S. A. Landsverk, A. Bernjak, A. Stefanovska, H. D. Kvernmo, and K. A. Kirkebøen, "Low-frequency oscillations of the laser Doppler perfusion signal in human skin," Microvascular Research, vol. 72, no. 3, pp. 120-127, 2006.

[6] G. Lancaster, A. Stefanovska, M. Pesce, G. Marco Vezzoni, B. Loggini, R. Pingitore, F. Ghiara, P. Barachini, G. Cervadoro, M. Romanelli, and M. Rossi, "Dynamic markers based on blood perfusion fluctuations for selecting skin melanocytic lesions for biopsy," Scientific Reports, vol. 5, p. 12825,2015

[7] A. I. Krupatkin, "Cardiac and respiratory oscillations of the blood flow in microvessels of the human skin," Human Physiology, vol. 34, no. 3, pp. 323-329, 2008.

[8] Z. Niazi, T. Essex, R. Papini, D. Scott, N. McLean, and M. Black, "New laser doppler scanner, a valuable adjunct in burn depth assessment," Burns, vol. 19, no. 6, pp. 485 - 489, 1993.

[9] K. Wardell, A. Jakobsson, and G. E. Nilsson, "Laser doppler perfusion imaging by dynamic light scattering," IEEE Transactions on Biomedical Engineering, vol. 40, no. 4, pp. 309-316, April 1993.

[10] M. Leutenegger, E. Martin-Williams, P. Harbi, T. Thacher, W. Raffoul, M. André, A. Lopez, P. Lasser, and T. Lasser, "Real-time full field laser Doppler imaging." Biomedical optics express, vol. 2, no. 6, pp. 1470-7, 2011.

[11] A. Serov and T. Lasser, "High-speed laser Doppler perfusion imaging using an integrating CMOS image sensor." Optics Express, vol. 13, no. 17 , pp. $6416-28,2005$.

[12] K. Basak, M. Manjunatha, and P. K. Dutta, "Review of laser specklebased analysis in medical imaging," Medical \& Biological Engineering \& Computing, vol. 50, no. 6, pp. 547-558, 2012.

[13] M. Draijer, E. Hondebrink, T. van Leeuwen, and W. Steenbergen, "Review of laser speckle contrast techniques for visualizing tissue perfusion," Lasers in Medical Science, vol. 24, no. 4, p. 639, 2008.

[14] M. Ansari and A. Nirala, "Monitoring capillary blood flow using laser speckle contrast analysis with spatial and temporal statistics," Optik, vol. 126 , no. 24 , pp. $5224-5229,2015$

[15] J. C. Ramirez-San-Juan, R. Ramos-Garcia, I. Guizar-Iturbide, G. Martinez-Niconoff, and B. Choi, "Impact of velocity distribution assumption on simplified laser speckle imaging equation," Optics Express, vol. 16, no. 5, pp. 3197-3203, 2008

[16] A. K. Dunn, "Laser speckle contrast imaging of cerebral blood flow," Annals of Biomedical Engineering, vol. 40, no. 2, pp. 367-377, 2012.

[17] I. Fredriksson and M. Larsson, "On the equivalence and differences between laser doppler flowmetry and laser speckle contrast analysis," Journal of Biomedical Optics, vol. 21, no. 12, p. 126018, 2016.

[18] M. J. Draijer, E. Hondebrink, M. Larsson, T. G. van Leeuwen, and W. Steenbergen, "Relation between the contrast in time integrated dynamic speckle patterns and the power spectral density of their temporal intensity fluctuations," Opt. Express, vol. 18, no. 21, pp. 21 883-21 891, 2010.

[19] O. B. Thompson and M. K. Andrews, "Tissue perfusion measurements: multiple-exposure laser speckle analysis generates laser doppler-like spectra," Journal of Biomedical Optics, vol. 15, no. 2, p. 027015, 2010.

[20] H. Furukawa and S. Hirotsu, "Dynamic light scattering from static and dynamic fluctuations in inhomogeneous media," Journal of the Physical Society of Japan, vol. 71, no. 12, pp. 2873-2880, 2002.

[21] G. Royl, C. Leithner, H. Sellien, J. P. Müller, and U. Lindauer, "Functional imaging with laser speckle contrast analysis: Vascular compartment analysis and correlation with laser doppler flowmetry and somatosensory evoked potentials," Brain Research, vol. 1121, pp. 95$103,2006$.

[22] T. Binzoni, A. HumeauHeurtier, P. Abraham, and G. Mah, "Blood perfusion values of laser speckle contrast imaging and laser doppler flowmetry: Is a direct comparison possible?" IEEE Transactions on Biomedical Engineering, vol. 60, no. 5, pp. 1259-1265, 2013.

[23] J. Zötterman, M. Bergkvist, F. Iredahl, E. Tesselaar, and S. Farnebo, "Monitoring of partial and full venous outflow obstruction in a porcine flap model using laser speckle contrast imaging," Journal of Plastic, Reconstructive \& Aesthetic Surgery, vol. 69, no. 7, pp. 936 - 943, 2016.

[24] A. K. Dunn, H. Bolay, M. A. Moskowitz, and D. A. Boas, "Dynamic imaging of cerebral blood flow using laser speckle." Journal of cerebral blood flow and metabolism : official journal of the International Society of Cerebral Blood Flow and Metabolism, vol. 21 3, pp. 195-201, 2001.

[25] D. Briers, D. Duncan, E. Hirst, S. Kirkpatrick, M. Larsson, W. Steenbergen, T. Stromberg, and O. Thompson, "Laser speckle contrast imaging: theoretical and practical limitations," Journal of Biomedical Optics, vol. 18, no. 6, p. 066018, 2013.

[26] A. Sdobnov, A. Bykov, G. Molodij, V. Kalchenko, T. Jarvinen, A. Popov, K. Kordas, and I. Meglinski, "Speckle dynamics under ergodicity 
breaking," Journal of Physics D: Applied Physics, vol. 51, no. 15, p. $155401,2018$.

[27] V. Kalchenko, D. Israeli, Y. Kuznetsov, and A. Harmelin, "Transcranial optical vascular imaging (TOVI) of cortical hemodynamics in mouse brain," Scientific Reports, vol. 4, no. 1, p. 5839, 2015.

[28] A. Y. Neganova, D. D. Postnov, O. Sosnovtseva, and J. C. B. Jacobsen, "Rat retinal vasomotion assessed by laser speckle imaging," PLOS ONE, vol. 12, no. 3, pp. 1-13, 2017.

[29] A. Humeau-Heurtier, P. Abraham, and G. Mahé, "Analysis of laser speckle contrast images variability using a novel empirical mode decomposition: Comparison of results with laser doppler flowmetry signals variability," IEEE Transactions on Medical Imaging, vol. 34, no. 2, pp. 618-627, 2015.

[30] A. Humeau-Heurtier, G. Mahe, S. Durand, and P. Abraham, "Skin perfusion evaluation between laser speckle contrast imaging and laser doppler flowmetry," Optics Communications, vol. 291, pp. 482-487, 2013.

[31] A. Humeau-Heurtier, B. Buard, G. Mahe, and P. Abraham, "Laser speckle contrast imaging of the skin: interest in processing the perfusion data," Medical \& Biological Engineering \& Computing, vol. 50, no. 2, pp. 103-105, 2012.

[32] M. D. Stern, "In vivo evaluation of microcirculation by coherent light scattering," Nature, vol. 254, pp. 56-58, 1975.

[33] P. Frick, I. Mizeva, and S. Podtaev, "Skin temperature variations as a tracer of microvessel tone," Biomedical Signal Processing and Control, vol. 21, pp. 1-7, 2015.

[34] S. J. Kirkpatrick, D. D. Duncan, and E. M. Wells-Gray, "Detrimental effects of speckle-pixel size matching in laser speckle contrast imaging," Opt. Lett., vol. 33, no. 24, pp. 2886-2888, Dec 2008.

[35] P. G. Vaz, A. Humeau-Heurtier, E. Figueiras, C. Correia, and J. Cardoso, "Laser speckle imaging to monitor microvascular blood flow: A review," IEEE Reviews in Biomedical Engineering, vol. 9, pp. 106-120, 2016.

[36] P. Goupillaud, A. Grossmann, and J. Morlet, "Cycle-octave and related transforms in seismic signal analysis," Geoexploration, vol. 23, no. 1, pp. $85-102,1984$.

[37] I. Mizeva, P. Frick, and S. Podtaev, "Relationship of oscillating and average components of laser doppler flowmetry signal," Journal of Biomedical Optics, vol. 21, no. 8, p. 085002, 2016.

[38] I. A. Mizeva, E. V. Potapova, V. V. Dremin, E. A. Zherebtsov, M. A. Mezentsev, V. V. Shupletsov, and A. V. Dunaev, "Optical probe pressure effects on cutaneous blood flow," Clinical Hemorheology and Microcirculation, vol. Pre-press, pp. 1-9, 2019.

[39] G. Mah, F. Haj-Yassin, P. Rousseau, A. Humeau, S. Durand, G. Leftheriotis, and P. Abraham, "Distance between laser head and skin does not influence skin blood flow values recorded by laser speckle imaging," Microvascular Research, vol. 82, no. 3, pp. 439 - 442, 2011.

[40] J. Zötterman, R. Mirdell, S. Horsten, S. Farnebo, and E. Tesselaar, "Methodological concerns with laser speckle contrast imaging in clinical evaluation of microcirculation," PLOS ONE, vol. 12, no. 3, pp. 1-11, 2017.

[41] A. Humeau-Heurtier, G. Mah, S. Durand, D. Henrion, and P. Abraham, "Laser speckle contrast imaging: Multifractal analysis of data recorded in healthy subjects," Medical Physics, vol. 39, no. 10, pp. 5849-5856, 2012.

[42] A. Stefanovska, M. Bracic, and H. D. Kvernmo, "Wavelet analysis of oscillations in the peripheral blood circulation measured by laser doppler technique," IEEE Transactions on Biomedical Engineering, vol. 46, pp. 1230-1239, 1999.

[43] I. Mizeva, E. Zharkikh, V. Dremin, E. Zherebtsov, I. Makovik, E. Potapova, and A. Dunaev, "Spectral analysis of the blood flow in the foot microvascular bed during thermal testing in patients with diabetes mellitus," Microvascular Research, vol. 120, pp. 13 - 20, 2018.

[44] J. O'Doherty, P. M. McNamara, N. Clancy, J. G. Enfield, and M. J. Leahy, "Comparison of instruments for investigation of microcirculatory blood flow and red blood cell concentration." Journal of biomedical optics, vol. 14 3, p. 034025, 2009.

[45] V. V. Dremin, E. Zherebtsov, V. V. Sidorov, A. I. Krupatkin, I. N. Makovik, A. I. Zherebtsova, E. V. Zharkikh, E. V. Potapova, A. V. Dunaev, A. A. Doronin, A. Bykov, I. E. Rafailov, K. S. Litvinova, S. G. Sokolovski, and E. U. Rafailov, "Multimodal optical measurement for study of lower limb tissue viability in patients with diabetes mellitus." Journal of biomedical optics, vol. 22 8, pp. 1-10, 2017. 